\title{
Light-Cone Effect and Supersonic Correlations in One and Two-Dimensional Bosonic Superfluids
}

\author{
Giuseppe Carleo, ${ }^{1}$ Federico Becca, ${ }^{2}$ Laurent Sanchez-Palencia, ${ }^{1}$ Sandro Sorella, ${ }^{2}$ and Michele Fabrizio ${ }^{2}$ \\ ${ }^{1}$ Laboratoire Charles Fabry, Institut d'Optique, CNRS, Univ. Paris Sud 11, \\ 2 avenue Augustin Fresnel, F-91127 Palaiseau cedex, France \\ ${ }^{2}$ Democritos Simulation Center CNR-IOM Istituto Officina dei Materiali and International \\ School for Advanced Studies (SISSA), Via Bonomea 265, 34136 Trieste, Italy
}

(Dated: March 28, 2014)

\begin{abstract}
We study the spreading of density-density correlations in Bose-Hubbard models after a quench of the interaction strength, using time-dependent variational Monte Carlo simulations. It gives access to unprecedented long propagation times and to dimensions higher than one. In both one and two dimensions, we find ballistic lightcone spreading of correlations and extract accurate values of the light-cone velocity in the superfluid regime. We show that the spreading of correlations is generally supersonic, with a light-cone propagating faster than sound modes but slower than the maximum group velocity of density excitations, except at the Mott transition, where all the characteristic velocities are equal. Further, we show that in two dimensions the correlation spreading is highly anisotropic and presents nontrivial interference effects.

PACS numbers: 05.30.Jp, 02.70.Ss, 03.75.Kk, 67.10.Jn
\end{abstract}

Introduction.- In 1972 Lieb and Robinson demonstrated that an effective light cone emerges in non-relativistic quantum many-body systems described by translation-invariant Hamiltonians, sums of finite-range interaction terms [1]. Specifically, they showed that any causal response function

$$
\chi_{A B}(\mathbf{r}, t)=-i\langle\Psi|[\mathscr{A}(\mathbf{r}, t), \mathscr{B}(\mathbf{0}, 0)]| \Psi\rangle,
$$

with $t>0$ and arbitrary $|\Psi\rangle$, decays exponentially for $|\mathbf{r}|>v t$ provided $\mathscr{A}(\mathbf{r}, t)$ and $\mathscr{B}(\mathbf{r}, t)$ are local operators in the Heisenberg form, i.e., such that $[\mathscr{A}(\mathbf{r}, t), \mathscr{B}(\mathbf{0}, t)]$ is non zero only for $\mathbf{r}=\mathbf{0}$. The velocity $v$ is finite and can be upper estimated by a properly defined operator norm of each local interaction term [1, 2]. The velocity $v$ does not depend on the wave function $|\Psi\rangle$, but only on the spectrum of the Hamiltonian. It is remarkable that, even though $|\Psi\rangle$ may be highly entangled and possess long-range correlations, any local perturbation needs a finite time to propagate up to a given distance. Such a locality principle constitutes a rather fundamental aspect in the dynamics of interacting many-body quantum systems, which is attracting considerable attention in recent years, mainly sparked by the impressive progress in ultracoldatom experiments. These experiments allow for a direct access to the non-equilibrium dynamics of relatively simple and quasi-isolated systems, making it possible to address issues that until recently were considered merely academic [3-5].

A related question arises when one considers instead equaltime correlations of the form

$$
N_{A B}(\mathbf{r}, t)=\langle\Psi|\mathscr{A}(\mathbf{r}, t) \mathscr{B}(\mathbf{0}, t)-\mathscr{A}(\mathbf{r}, 0) \mathscr{B}(\mathbf{0}, 0)| \Psi\rangle .
$$

Although in $N_{A B}(\mathbf{r}, t)$ the measurement is instantaneous, unlike in $\chi_{A B}(\mathbf{r}, t)$, several arguments suggest that an horizon effect emerges even for $N_{A B}(\mathbf{r}, t)$, with a light-cone velocity twice as large as the Lieb-Robinson bound [6, 7]. Early evidence of a light-cone effect in the dynamics induced by interaction quenches in one-dimensional Bose-Hubbard models was found in Ref. [8] using time-dependent density-matrix renormalization group (tDMRG) [9, 10] and confirmed experimentally in Ref. [11]. However, these first results raise intriguing questions that are worth investigating. On the one hand, outside the Mott insulator phase, the bosons form a superfluid with power-law correlations. The infinite correlation length, alike a system right at criticality, would suggest that the light-cone velocity is just once or twice the sound velocity (i.e., the velocity of the critical modes) for the correlation functions (11) and (2) respectively, as predicted by conformalfield theory (CFT) [7]. The t-DMRG analysis appears to call into question the CFT prediction [8]. However, accurate determination of the propagation velocity and comparison to the characteristic velocities of the system remain open questions. On the other hand, the spreading of correlations in dimensions higher than one constitutes an almost unexplored land, where t-DMRG approaches do not apply. This question is particularly relevant in view of the possibility of extending the experimental results [11, 12] in higher dimensions.

In this paper we study these questions using the recently introduced time-dependent Variational Monte Carlo (t-VMC) approach [13], which allows us to address asymptotically long propagation times and dimensions higher than one. Specifically we study the spreading of density-density correlations after a quench in the interaction strength of the Bose-Hubbard model in one (1D) and two (2D) dimensions. For both cases in the superfluid regime, we find a supersonic light-cone effect. More precisely, we find that the light-cone velocity differs from both twice the sound velocity and twice the maximum excitation velocity, except when approaching the Mott transition, where these velocities are equal. Moreover, we show that in 2D the correlation spreading is highly anisotropic and present nontrivial interference effects. The anisotropy of the correlation front is however simply explained in terms of the lattice coordination within the Manhattan metrics.

System and method.- We consider non-relativistic lattice 
bosons described by the Bose-Hubbard Hamiltonian

$$
\mathscr{H}(U)=-\sum_{\left\langle\mathbf{R}, \mathbf{R}^{\prime}\right\rangle}\left(b_{\mathbf{R}}^{\dagger} b_{\mathbf{R}^{\prime}}+\text { h.c. }\right)+\frac{U}{2} \sum_{\mathbf{R}} n_{\mathbf{R}}\left(n_{\mathbf{R}}-1\right),
$$

where $\mathbf{R}$ denotes a lattice site, $\left\langle\mathbf{R}, \mathbf{R}^{\prime}\right\rangle$ a pair of nearestneighbor sites, $b_{\mathbf{R}}^{\dagger}\left(b_{\mathbf{R}}\right)$ the creation (annihilation) operator of a boson on site $\mathbf{R}, n_{\mathbf{R}}=b_{\mathbf{R}}^{\dagger} b_{\mathbf{R}}$ the boson density on site $\mathbf{R}$, and $U$ the two-body interaction strength. In the following, the lattice will be either a $1 \mathrm{D}$ chain or a $2 \mathrm{D}$ square lattice, with periodic boundary conditions and average density $\left\langle n_{\mathbf{R}}\right\rangle=1$. The system is first prepared in the ground state of $\mathscr{H}\left(U_{\mathrm{i}}\right)$. At time $t=0$, it is then driven out of equilibrium upon realizing a sudden quantum quench in the interaction strength, from $U_{\mathrm{i}}$ to $U_{\mathrm{f}}$. We study the dynamics of the density-density correlation function

$$
N(\mathbf{R}, t)=\left\langle n_{\mathbf{R}}(t) n_{\mathbf{0}}(t)\right\rangle-\left\langle n_{\mathbf{R}}(0) n_{\mathbf{0}}(0)\right\rangle,
$$

where the average is over the ground state of $\mathscr{H}\left(U_{\mathrm{i}}\right)$ and the density operators are evolved in time with $\mathscr{H}\left(U_{\mathrm{f}}\right)$ i.e., Eq. (2) where both $\mathscr{A}$ and $\mathscr{B}$ are the density operators.

Our analysis makes use of the t-VMC approach [13] that we briefly outline here. The starting point is to define a class of time-dependent variational many-body wave functions, which we take of the Jastrow type

$$
\Psi(\mathbf{x}, t) \equiv\langle\mathbf{x} \mid \Psi(t)\rangle=\exp \left[\sum_{r} \alpha_{r}(t) \mathscr{O}_{r}(\mathbf{x})\right] \Phi_{0}(\mathbf{x}),
$$

where $\mathbf{x}$ spans a configuration basis, $\Phi_{0}(\mathbf{x})$ is a bosonic timeindependent state, and $\alpha_{r}(t)$ are complex variational parameters coupled to a set of operators $\mathscr{O}_{r}$ that are diagonal in the $\mathbf{x}$ basis, i.e., $\left\langle\mathbf{x}\left|\mathscr{O}_{r}\right| \mathbf{x}^{\prime}\right\rangle=\delta_{\mathbf{x}, \mathbf{x}^{\prime}} \mathscr{O}_{r}(\mathbf{x})$. The explicit form of these operators and their total number define the variational subspace. Here we use the Fock basis, $\mathbf{x}=\left\{n_{i}\right\}$, and the complete set of density-density correlations, $\mathscr{O}_{\mathbf{r}}=\sum_{\mathbf{R}} n_{\mathbf{R}} n_{\mathbf{R}+\mathbf{r}}$, where $\mathbf{r}$ spans all independent distances on the lattice. The initial state is chosen to be the variational Jastrow ground state of $\mathscr{H}\left(U_{\mathrm{i}}\right)$ with $\left|\Phi_{0}\right\rangle$ the noninteracting-boson ground state of $\mathscr{H}(0)$. This choice provides an excellent approximation of the exact ground state of $\mathscr{H}\left(U_{\mathrm{i}}\right)$ [14, 15]. For instance, the superfluid-insulator transition is obtained for $U_{\mathrm{c}}^{\mathrm{var}} \simeq 5$ and $U_{\mathrm{c}}^{\mathrm{var}} \simeq 21$ in $1 \mathrm{D}$ and $2 \mathrm{D}$ respectively, in fair agreement with exact results [16, 17].

The variational dynamics of the system is fully contained in the trajectories of the variational parameters $\alpha_{r}(t)$. The latter are obtained by minimizing the Hilbert-space distance between the infinitesimal exact dynamics and the time derivative of the variational state (5) at each time step. This process is equivalent to project the exact time-evolved wave function onto the variational subspace. It yields a closed set of coupled equations of motion:

$$
i \sum_{r^{\prime}} S_{r, r^{\prime}}(t) \dot{\alpha}_{r^{\prime}}(t)=\left\langle\mathscr{O}_{r} \mathscr{H}\right\rangle_{t}-\left\langle\mathscr{O}_{r}\right\rangle_{t}\langle\mathscr{H}\rangle_{t}
$$

where $S_{r, r^{\prime}}(t)=\left\langle\mathscr{O}_{r} \mathscr{O}_{r^{\prime}}\right\rangle_{t}-\left\langle\mathscr{O}_{r}\right\rangle_{t}\left\langle\mathscr{O}_{r^{\prime}}\right\rangle_{t}$ and the quantum averages are taken over the time-dependent variational state (5).
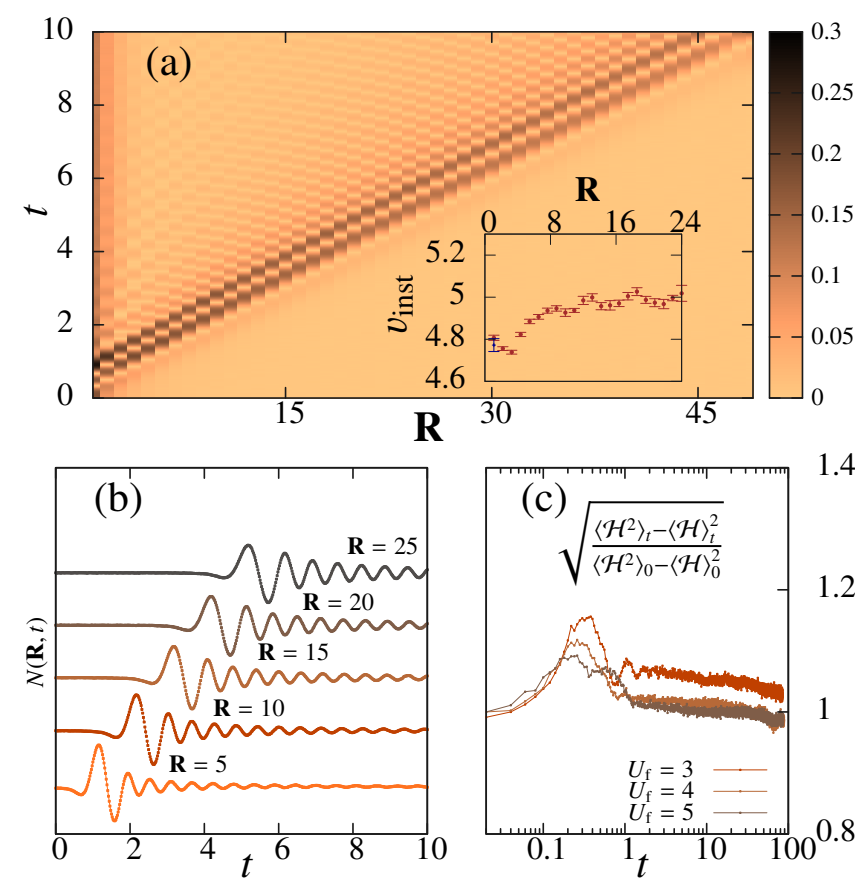

Figure 1: (Color on-line) Spreading of correlations in a 1D chain. (a) Density-density correlations $N(R, t)$ versus separation and time for a quench in the interaction strength from $U_{\mathrm{i}}=2$ to $U_{\mathrm{f}}=4$. The inset shows the instantaneous velocity as obtained from t-VMC (red points) and exact diagonalization (for a 12-site lattice; blue point). (b) Time dependence of $N(R, t)$ for various values of $R$. For clarity, the curves are vertically shifted by a value proportional to $R$, and the linear light-cone wave-front clearly appears. (c) Relative energy fluctuations versus time for various values of $U_{\mathrm{f}}$. The $\mathrm{t}-\mathrm{VMC}$ calculations are performed for 200 (a and b) or 500 (c) sites.

At each time, the quantum averages appearing in Eq. (6) are computed by variational Monte Carlo simulations and the linear system of equations (6) is solved for $\dot{\alpha}_{r}(t)$. The trajectories $\alpha_{r}(t)$ are then found by time-integrating the functions $\dot{\alpha}_{r}(t)$.

We emphasize that our variational scheme is symplectic and exactly conserves both the total energy and the square modulus of the wave function. In the numerical calculations, we use a sufficiently small time-step, $\delta t=0.01$, and a fourth-order Runge-Kutta integration scheme, which conserves the energy with a very small systematic error of the order of one part in a thousand, for times up to $t=100$. The t-VMC is therefore intrinsically stable, amenable to simulating time scales that exceed by about two orders of magnitudes those achievable by t-DMRG in 1D, and applies as well in higher dimensions.

Results. - Let us first discuss our results for the 1D chain. Figure 1 a) shows the density-density correlation $N(R, t)$ as a function of separation and time for a quantum quench from $U_{\mathrm{i}}=2$ to $U_{\mathrm{f}}=4$. Figure 1 b) shows vertical cuts of the latter, plotted with a vertical shift proportional to $R$ for clarity. A light-cone effect is clearly visible: $N(R, t)$ is unaffected at short times, then develops a maximum at a finite time $t^{\star}(R)$, and finally undergoes damped oscillations. Similar results are 
found for all quenches discussed below. For large enough separation, the activation time $t^{\star}(R)$ depends linearly on the separation, $t^{\star}(R) \equiv v_{\mathrm{lc}} \times R$, which defines the light-cone velocity $v_{\text {lc }}$. More precisely, the instantaneous correlation-spreading velocity, $v_{\text {inst }}(R) \equiv \frac{2}{t^{\star}(R+1)-t^{\star}(R-1)}$, is shown as a function of $R$ in the inset of Fig. 1 1 a). The ballistic regime, where $v_{\text {inst }}(R)$ approaches $v_{\mathrm{lc}}$, is achieved only for sufficiently long time $\left(t_{\text {ball }} \sim 4\right)$. The t-VMC method allows us to simulate very long times in the asymptotic ballistic regime $(t \sim 100)$, and extract accurate values of $v_{\mathrm{lc}}$.

At variance with the total energy, higher moments of the Hamiltonian are not strictly conserved by the t-VMC scheme, as illustrated in Fig. 1. (c). Nevertheless, despite a slight timedependence of the energy fluctuations at very short times, the long-time value always coincides with the initial value, showing the accuracy of our variational method. In order to further check it, we compared our results (red points) to exact diagonalization (blue point) at time $t \simeq 0.5$ close to the maximal deviation of the energy fluctuations (inset of Fig. 11(a)). We found very good agreement, hence confirming the accuracy of t-VMC [28].

The very existence of a finite propagation velocity and its microscopic origin can be justified as follows. Assume $|n\rangle$ and $|m\rangle$ are two eigenstates of $\mathscr{H}\left(U_{\mathrm{f}}\right)$ with eigenvalues $E_{l}$ and $E_{m}$, and total momentum $\mathbf{P}+\mathbf{q}$ and $\mathbf{P}$, respectively, such that $\left\langle l\left|\mathscr{A}_{\mathbf{q}}\right| m\right\rangle$, with $\mathscr{A}_{\mathbf{q}}=\sum_{\mathbf{r}} \mathscr{A}(\mathbf{r}) \mathrm{e}^{i \mathbf{q} \cdot \mathbf{r}}$, is finite. If $\mathscr{A}(\mathbf{r})$ is a bounded local operator, then $\omega_{l m}(\mathbf{P}, \mathbf{q})=E_{l}-E_{m}$ is not an extensive quantity, though $E_{l}$ and $E_{m}$ are both extensive. For large $\mathbf{r}$, i.e. small $\mathbf{q}$, such excitation can propagate coherently only if $|\mathbf{r}| \simeq t\left|\partial_{\mathbf{q}} \omega_{l m}(\mathbf{P}, \mathbf{0})\right|$. This defines a maximum propagation velocity $v_{\mathrm{m}} \equiv \operatorname{Max}\left|\partial_{\mathbf{q}} \omega_{l m}(\mathbf{P}, \mathbf{0})\right|$ to be identified with the Lieb-Robinson bound. In the case of Eq. (2), if $|\Psi\rangle$ is an eigenstate of defined total momentum, then two counterpropagating excitations are involved due to momentum conservation, and the bound velocity is $2 v_{\mathrm{m}}$. For small quenches towards a gapless phase, one may expect that only low-energy phonon excitations are involved, and that the light-cone velocity is twice the sound velocity, $2 v_{\mathrm{s}}$.

The value of $v_{\text {lc }}$ is plotted in Fig. 2 (a) as a function of the final interaction strength $U_{\mathrm{f}}$ for various values of initial interaction strength $U_{\mathrm{i}}$. We find that $v_{\mathrm{lc}}$ increases with $U_{\mathrm{f}}$, which is readily understood by the fact that the rigidity of the final lattice increases with $U_{\mathrm{f}}$. It is remarkable however that $v_{\mathrm{lc}}$ does not depend on $U_{\mathrm{i}}$. In Fig. 2, the t-VMC value for $v_{\mathrm{lc}}$ is compared to the characteristic velocities of the density excitations, i.e. $2 v_{\mathrm{m}}$ and $2 v_{\mathrm{s}}$. The latter ones are computed as $v_{\mathrm{m}}=$ $\max \{\partial E(q) / \partial q\}$ and $v_{\mathrm{s}}=\lim _{q \rightarrow 0} \partial E(q) / \partial q$, where $E(q)$ is the energy of the density modes $|\psi(q)\rangle=\rho(q)\left|\psi_{0}\right\rangle$, with $\rho(q)$ the Fourier transform of the density operator [14, 18]. We generically find that the light-cone velocity significantly differs from twice both these velocities. On the one hand, the maximum velocity allowed by the propagation of excitations is not achieved, in contrast to quenches from the Mott phase [11, 20]. On the other hand, a supersonic regime is achieved in all the superfluid region of the out-of-equilibrium phase diagram, even for very small quenches. For instance,
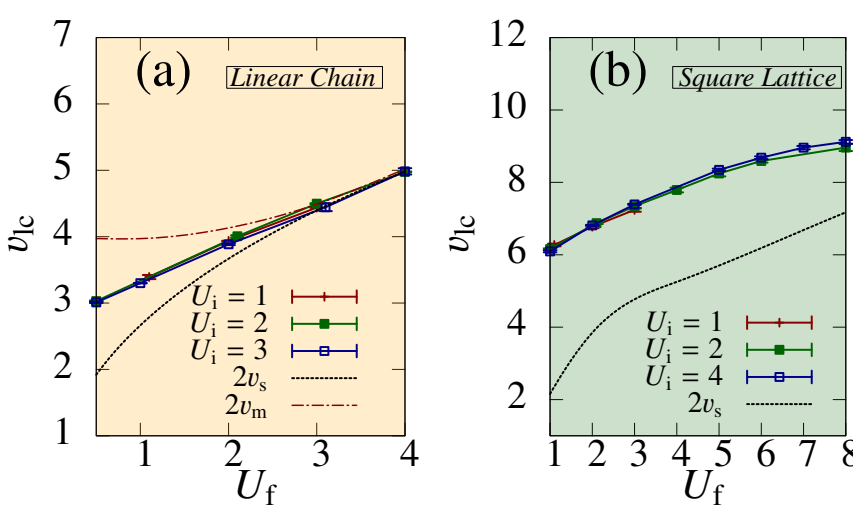

Figure 2: (Color on-line) Light-cone velocity $v_{\mathrm{lc}}$ versus the final interaction strength $U_{\mathrm{f}}$, for various values of the initial interaction strength $U_{\mathrm{i}}$. (a) $1 \mathrm{D}$ chain. (b) $2 \mathrm{D}$ square lattice. Also shown are twice the sound velocity, $2 v_{\mathrm{s}}$, and twice the maximum excitation velocity, $2 v_{\mathrm{m}}$, for $\mathscr{H}\left(U_{\mathrm{f}}\right)$.

in the case of a quench from $U_{\mathrm{i}}=1$ to $U_{\mathrm{f}}=1.1$, we find $v_{\mathrm{lc}}=3.39(3)$ and $2 v_{\mathrm{s}}=2.78$ [see Fig. 2(a)]. Therefore, highenergy excitations beyond the sound-wave regime are always generated by the quench dynamics. It corresponds to shortdistance effects that are always significant but not accounted for in CFT [7]. A form of universality is recovered only in the neighborhood of the Mott transition. When the final interaction strength approaches the critical value, $U_{\mathrm{c}}^{\mathrm{var}} \simeq 5$ at the variational level, the excitation modes exhibit a maximal velocity at zero momentum and all the characteristic velocities, $v_{\mathrm{lc}}, 2 v_{\mathrm{m}}$, and $2 v_{\mathrm{s}}$ coincide. It suggests that the results of CFT are correct only when the quantum quench is performed right at a critical point and not in the whole quasi-long-range ordered phase with infinite correlation length, i.e., for $U_{\mathrm{f}}<U_{\mathrm{c}}^{\mathrm{var}}$.

We now turn to the $2 \mathrm{D}$ square lattice. The spreading of correlations in dimension higher than one constitutes an almost unexplored land where only mean-field methods have been applied so far [21-26]. The latter are reliable only in the unphysical limits of large lattice connectivity or large internal "flavor" degeneracy. In contrast, t-VMC takes into account relevant dynamical correlations and can be applied to the physical Bose-Hubbard Hamiltonian in any dimension. Figure 3 (a) shows the correlation function $N(\mathbf{R}, t)$ at equally separated times, for a quench from $U_{\mathrm{i}}=2$ to $U_{\mathrm{f}}=4$ in the $2 \mathrm{D}$ square lattice. It shows a clear spreading of correlations. The correlation front is a square with principal axes along the diagonals of the lattice. In order to understand this, notice that nearest-neighbor hopping in the square lattice induces a natural metrics that is of the Manhattan type [27], rather than Euclidean. Points at equal Manhattan distance $d_{\operatorname{man}}(\mathbf{R}) \equiv$ $\left|R_{x}\right|+\left|R_{y}\right|$ are thus located on $45^{\circ}$-tilted squares. Figure 3 b) shows the activation time $t^{\star}(\mathbf{R})$, defined as the time when the first maximum of $N(\mathbf{R}, t)$ appears, versus the Manhattan distance for various lattice sites. The data for various $\mathbf{R}$ but same $d_{\text {man }}(\mathbf{R})$ collapse, which confirms that the Manhattan distance is the relevant metrics. Moreover, within the Man- 

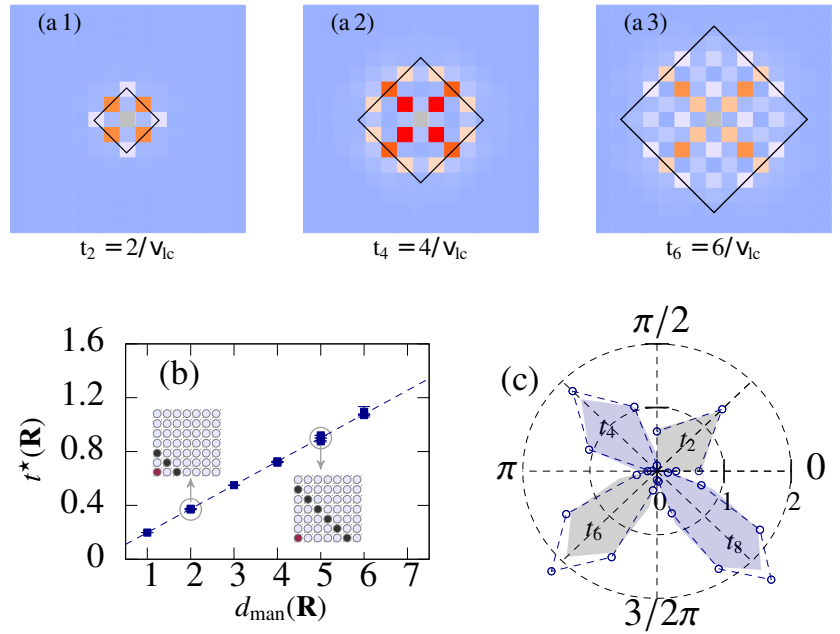

Figure 3: (Color on-line) Spreading of correlations in a $20 \times 20$ site square lattice for a quench from $U_{\mathrm{i}}=2$ to $U_{\mathrm{f}}=4$. (a) Densitydensity correlations $N(\mathbf{R}, t)$ at fixed times $t_{n}=n / v_{\mathrm{lc}}$. The $45^{\circ}$-tilted squares denote the points on the correlation front. (b) Activation time $t^{\star}(\mathbf{R})$ versus Manhattan distance $d_{\operatorname{man}}(\mathbf{R})$ for various points $\mathbf{R}$. The insets show the ensemble of points $\mathbf{R}$ with equal $d_{\operatorname{man}}(\mathbf{R})$ at the corresponding Manhattan distance. The dashed line is a linear fit to the data. (c) Intensity of the correlation signal (dots) and number of paths (shaded areas) versus the azimuthal angle of the points on correlation fronts. Each quadrant corresponds to a polar plot at the 4 different times indicated on the Figure.

hattan metrics, a clear ballistic behavior is observed, which allows us to define the light-cone velocity $v_{\mathrm{lc}} \equiv d_{\operatorname{man}}(\mathbf{R}) / t^{\star}(\mathbf{R})$. In Fig. 2(b), we show the extracted values of $v_{\mathrm{lc}}$ as a function of $U_{\mathrm{f}}$ for various values of $U_{\mathrm{i}}$, together with twice the sound velocity for the $2 \mathrm{D}$ square lattice. As for the $1 \mathrm{D}$ chain, a strong discrepancy between these two velocities is found also in 2D. This outcome indicates that high-energy excitations dominate the dynamical evolution even for small quenches, although the initial state is genuinely off-diagonal long-range ordered. It contrasts with low-enegy descriptions that take into account only sound modes.

As it can be seen on Fig. 3. a), the correlation signal shows complicated, anisotropic patterns, as a result of nontrivial interference effects. For instance at variance with the 1D case, the time-dependence of $N(\mathbf{R}, t)$ can show several secondary maxima with a stronger amplitude than the wavefront. The anisotropy can however be understood on the wavefront where the interference effects are weak. Indeed, two points $(0,0)$ and $\mathbf{R}=\left(R_{x}, R_{y}\right)$ are generically connected by a number $N_{\operatorname{man}}(\mathbf{R}, d)$ of paths of total length $d$, which do not depend only on $d_{\operatorname{man}}(\mathbf{R})$. On the wavefront, $d=d_{\operatorname{man}}(\mathbf{R})$ and $N_{\operatorname{man}}[\mathbf{R}, d]=\left(\left|R_{x}\right|+\left|R_{y}\right|\right) ! /\left|R_{x}\right| !\left|R_{y}\right| !$, which grows from 1 on the angles to $d ! /[(d / 2) !]^{2}$ on the center of the sides. This explains that the maxima are located on the main axis of the correlation square. More precisely, Fig. 3 (c) shows both the intensity of the correlation signal (points) and the number of connecting paths $N_{\operatorname{man}}[\mathbf{R}, d]$ (shaded areas), for various times and various points on the wavefront. The quantitative agreement between the two confirms that the main source of anisotropy on the correlation front is geometrical.

Conclusions. - We have studied the spreading of densitydensity correlations after a quantum quench in 1D and 2D Bose-Hubbard models, using the recently developed t-VMC approach. Our results show a light-cone ballistic expansion of correlations in both cases, and provide accurate values of the light-cone velocity. Our main result is that the light-cone velocity significantly differs from both twice the sound velocity and twice the maximum excitation velocity, except when approaching the Mott transition. Moreover, in 2D, the correlation signal is highly anisotropic and the correlation front is a square, which is due to the Manhattan metrics imposed by the nearest-neighbor lattice coordination. Our results provide new insight on the spreading of correlations in interacting quantum systems. They also offer an important benchmark for future experiments with ultracold atomic gases in optical lattices, especially in dimension higher than one.

We acknowledge discussions with I. Bouchoule, I. Carusotto, M. Cheneau, and M. Schiro. This research was supported by the European Research Council (FP7/2007-2013 Grant Agreement No. 256294), Marie Curie IEF (FP7/20072013 - Grant Agreement No. 327143) and PRIN 2010-11. Use of the computing facility cluster GMPCS of the LUMAT federation (FR LUMAT 2764) is acknowledged.

[1] E.H. Lieb and D.W. Robinson, Commun. Math. Phys. 28, 251 (1972).

[2] B. Nachtergaele and R. Sims, Commun. Math. Phys. 265, 119 (2006).

[3] A. Polkovnikov, K. Sengupta, A. Silva, and M. Vengalattore, Rev. Mod. Phys. 83, 863 (2011).

[4] M. Lewenstein, A. Sanpera, V. Ahufinger, B. Damski, A. Sen De, and U. Sen, Adv. Phys. 56243 (2007).

[5] I. Bloch, J. Dalibard, and W. Zwerger, Rev. Mod. Phys. 80, 885 (2008).

[6] S. Bravyi, M.B. Hastings, and F. Verstraete, Phys. Rev. Lett. 97, 050401 (2006).

[7] P. Calabrese and J. Cardy, Phys. Rev. Lett. 96, 136801 (2006); J. Stat. Mech. P06008 (2007).

[8] A.M. Lauchli and C. Kollath, J. Stat. Mech. P05018 (2008).

[9] S.R. White and A.E. Feiguin, Phys. Rev. Lett. 93, 076401 (2004).

[10] A.J. Daley, C. Kollath, U. Schollwoeck, and G. Vidal, J. Stat. Mech. P04005 (2004).

[11] M. Cheneau, P. Barmettler, D. Poletti, M. Endres, P. Schauss, T. Fukuhara, C. Gross, I. Bloch, C. Kollath, and S. Kuhr, Nature 481, 484 (2012).

[12] T. Langen, R. Geiger, M. Kuhnert, B. Rauer, and J. Schmiedmayer, Nat. Phys. 9, 640 (2013).

[13] G. Carleo, F. Becca, M. Schiro, and M. Fabrizio, Sci. Rep. 2, 243 (2012).

[14] M. Capello, F. Becca, M. Fabrizio, and S. Sorella, Phys. Rev. Lett. 99, 056402 (2007). 
[15] M. Capello, F. Becca, M. Fabrizio, and S. Sorella, Phys. Rev. B 77, 144517 (2008).

[16] T.D. Kuhner and H. Monien, Phys. Rev. B 58, 14741 (1998).

[17] B. Capogrosso-Sansone, S.G. Soyler, N. Prokof'ev, and B. Svistunov, Phys. Rev. A 77, 015602 (2008).

[18] The sound velocity may be computed by considering the size corrections of the ground-state energy. [15] In one dimension, the Lieb-Liniger result $v_{L L}=2 \sqrt{\gamma-\gamma^{3 / 2} / 2 \pi}$, with $\gamma=U / 2 J$, [19] gives an excellent approximation to the sound velocity.

[19] E.H. Lieb, Phys. Rev. 130, 1616 (1963).

[20] P. Barmettler, D. Poletti, M. Cheneau, and C. Kollath, Phys. Rev. A 85, 053625 (2012).

[21] C. Menotti, C. Trefzger, and M. Lewenstein, Phys. Rev. Lett. 98, 235301 (2007).
[22] S. Sotiriadis and J. Cardy, Phys. Rev. B 81, 134305 (2010).

[23] B. Sciolla and G. Biroli, Phys. Rev. Lett. 105, 220401 (2010).

[24] M. Schiro and M. Fabrizio, Phys. Rev. Lett. 105, 076401 (2010).

[25] I. Carusotto, R. Balbinot, A. Fabri and A. Recati, Eur. Phys. J. D 56, 391 (2010).

[26] S.S. Natu, and E.J. Mueller, Phys. Rev. A 87, 063616 (2013).

[27] E. F. Krause, Taxicab Geometry, (Courier Dover Publications, 1986).

[28] In addition, we have compared the t-VMC velocities with the ones obtained by t-DMRG in Ref.[8]. The two results are in quantitative agreement, within the error bars of the t-dmrg calculation. 